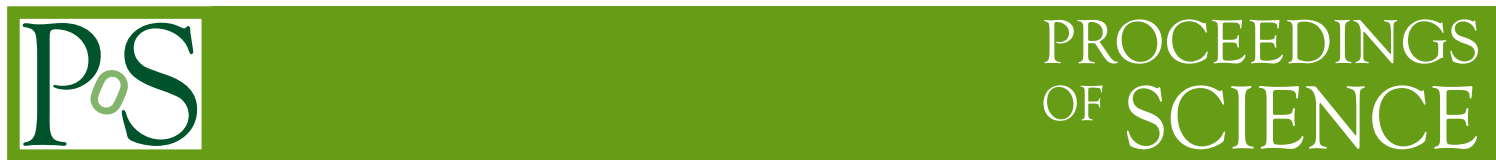

\title{
Deuteron Compton Scattering Above Pion Threshold
}

\author{
L.S. Myers ${ }^{* \dagger}$ \\ Bluffton University \\ E-mail: myersl@bluffton.edu
}

Results are reported of the first experimental measurement of deuteron Compton scattering cross sections at photon energies above pion threshold. The measurements are not precise enough to provide a meaningful extraction of the electromagnetic polarizabilities. However, this experiment demonstrates that measurements above pion threshold are feasible. As a results, future versions of this measurement could be used to improve the current state of deuteron Compton scattering data set and the extracted neutron polarizabilities.

The 9th International workshop on Chiral Dynamics

17-21 September 2018

Durham, NC, USA

\footnotetext{
* Speaker.

†Presented on behalf of the COMPTON@MAX-lab collaboration.
} 


\section{Motivation}

This work [1] represents a continuation of the study of the electric and magnetic polarizabilities ( $\alpha_{n}$ and $\beta_{n}$, respectively) of the nucleon. There has been much progress in the experimental study of the nucleon static and spin polarizabilities over the past few decades. See References citeGri12 and [3] for review articles of prior experiments. Currently, the static polarizabilities of the proton are known more precisely than those of the neutron, as seen in Table 1 where the values are taken from [4]. This discrepancy has implications for both the study of the polarizabilities (it is more difficult to extract the spin polarizabilities when the static polarizabilities are poorly constrained) as well as other topics in nuclear physics such as calculations of the proton-neutron mass difference [5] and the Lamb shift of muonic hydrogen [6]. In order to improve the uncertainties of the neutron electromagnetic polarizabilities more experimental data is required. Additional data should increase the kinematical range of the existing data, including an extension to energies above pion photoproduction threshold that would balance the neutron and proton world data sets. The experiment detailed below sets out to establish the feasibility of a measurement taken at photon energies at and just above pion photoproduction threshold.

\begin{tabular}{ccc} 
& Proton $\left[10^{-4} \mathrm{fm}^{3}\right]$ & Neutron $\left[10^{-4} \mathrm{fm}^{3}\right]$ \\
\hline$\alpha$ & $10.65 \pm 0.35_{\text {stat }} \pm 0.2_{B S R} \pm 0.3_{\text {th }}$ & $11.55 \pm 1.25_{\text {stat }} \pm 0.2_{B S R} \pm 0.8_{\text {th }}$ \\
$\beta$ & $3.15 \mp 0.35_{\text {stat }} \pm 0.2_{B S R} \mp 0.3_{\text {th }}$ & $3.65 \mp 1.25_{\text {stat }} \pm 0.2_{B S R} \mp 0.8_{\text {th }}$
\end{tabular}

Table 1: Summary of recent extractions of the electric $(\alpha)$ and magnetic $(\beta)$ polarizabilities with statistical, theoretical, and Baldin-sum-rule uncertainties. Taken from [4].

\section{Experiment}

This measurement was conducted at the former Tagged Photon Facility (TPC) at the MAX IV laboratory in Lund, Sweden[7]. The pertinent details of the experiment are described below; the reader is referred to [1] for additional information.

A 190-MeV energy electron beam was injected into the TPC where it was incident upon a thin aluminum foil. The recoil electrons were momentum-analyzed using the SAL tagger magnet and hodoscope $[8,9]$. The bremsstrahlung photons were collimated and then fell upon a 17-cmlong liquid deuterium target. Photons that Compton-scattered from the target were detected by one of the three large-volume $\mathrm{NaI}$ detectors placed at $60^{\circ}, 120^{\circ}$, and $150^{\circ}$. Coincidences between a photon in the $\mathrm{NaI}$ detector and an electron in the hodoscope are used to determine the photon energy. For this measurement, the tagged photon energies are in the nominal energy range of 140-160 MeV. Each detector has an energy resolution of $\Delta E \leq 2 \%$ at the tagged energies. The experimental setup is shown in Figure 1.

The data collected for this measurement were obtained simultaneously to data for a measurement of $\pi^{-}$photoproduction off the neutron in three run periods in 2012 and 2015. Due to the priority of the $\pi^{-}$measurement, the run conditions were not optimized for extracting a Comptonscattering cross section. The goal was to demonstrate the feasibility of measuring the $d\left(\gamma, \gamma^{\prime}\right) d$ cross section above pion threshold - the first measurement of its kind. 


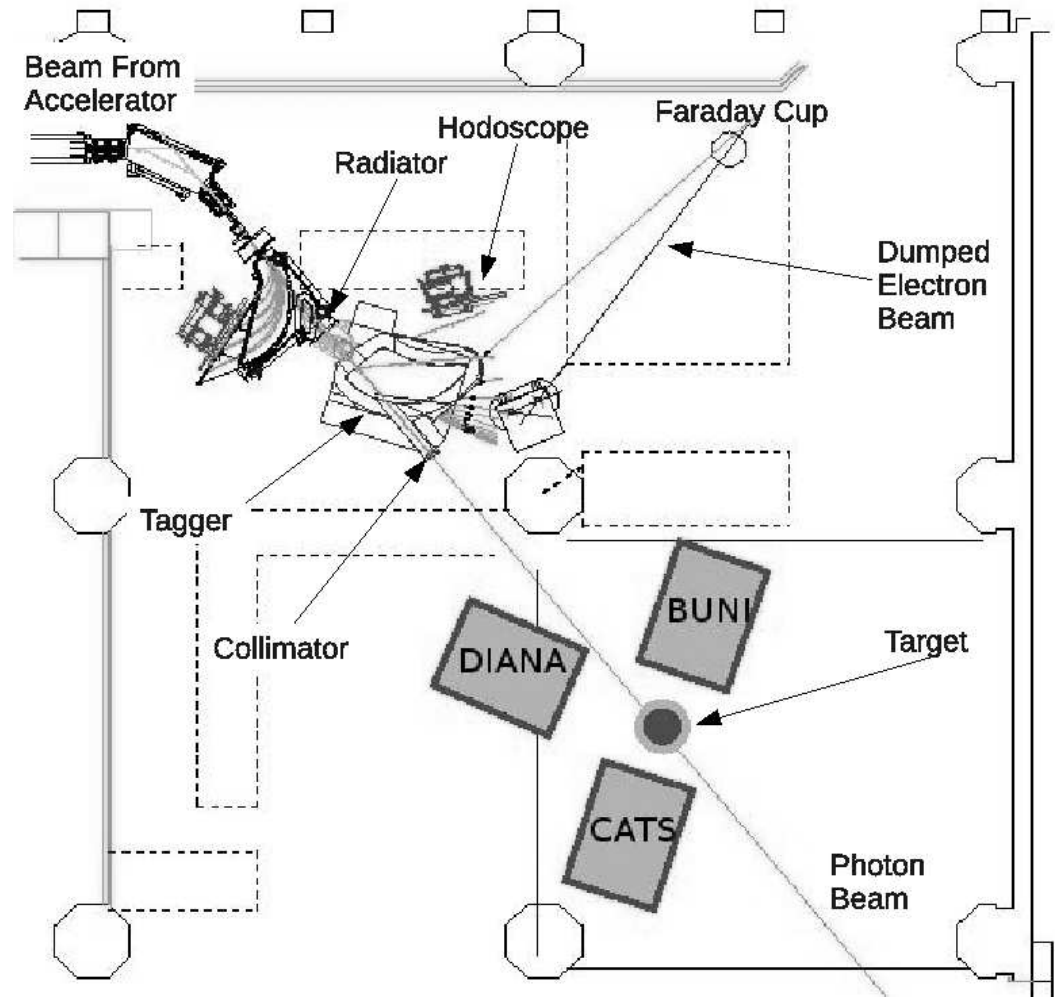

Figure 1: Experimental layout showing the locations of the focal plane, target and $\mathrm{NaI}$ detectors for the Compton scattering experiments.

The new challenge facing near-threshold measurements of deuteron Compton scattering is the contamination from photon-producing reactions involving the pions. The large pion photoproduction cross section $(\sim 100 \times$ greater than the Compton cross section) indicates that photons emitted from, for example, the $\pi^{-}+d \rightarrow 2 n+\gamma$ reaction, can overwhelm the elastically scattered photons. Plotting the photon energies from Compton scattering off the deuteron at the detector angles (see Figure 2), and taking a cutoff of $E_{\text {thresh }} \approx 135 \mathrm{MeV}$ to eliminate contribution from the capture photons, already demonstrates that the best-case scenario for this experiment is to collect a full range (four 5-MeV-wide bins) of data at $60^{\circ}$, and possibly one or two bins at the back angles.

\section{Analysis and Results}

The data were analyzed by identifying photons whose energy fell within a narrow interval of the tagged photon energy $\left(E_{\text {miss }}=E_{\text {det }}-E_{\gamma}^{\prime} \in[-2,3] \mathrm{MeV}\right)$. The timing spectra of these photons was produced to identify the coincident peak, which was integrated to obtain the photon yield. (See Figure 3 [1].) The photon yield is then normalized to the number of incident photons, target thickness, and detector solid angle to obtain the scattering cross section. A small corrections were applied to account for absorption of photons in the target. Unlike previous Compton-scattering work at MAX IV, the recoil electron rate in the hodoscope channels was low enough $(100 \mathrm{kHz}$ vs $1 \mathrm{MHz}$ ) that rate-dependent effects were minimal. 


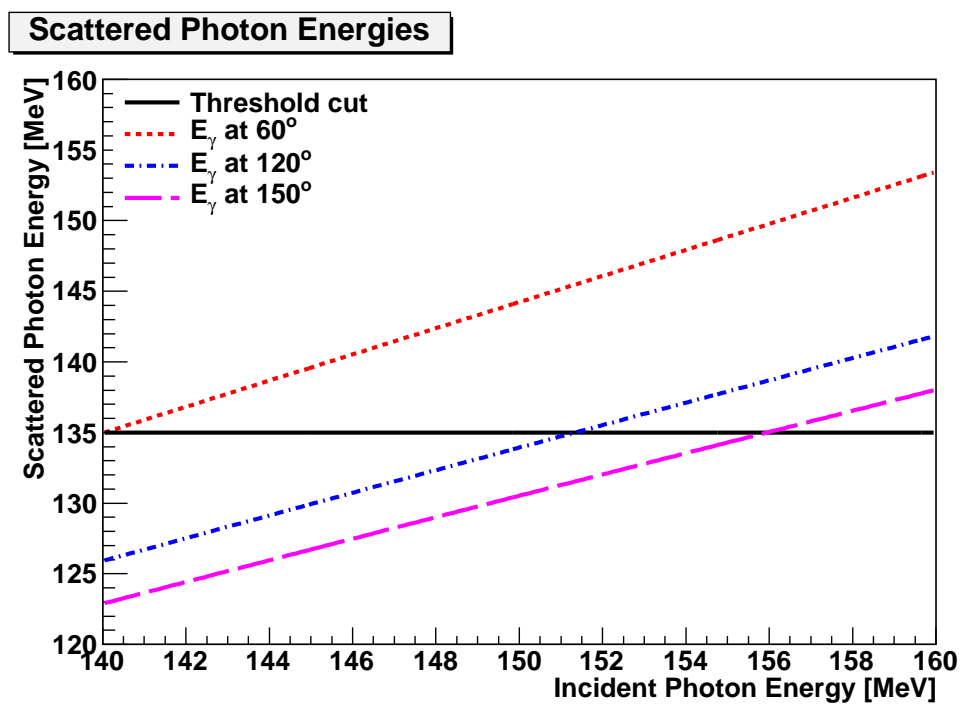

Figure 2: Compton-scattered photon energies at each detector angle. The threshold cut at $135 \mathrm{MeV}$ (to remove contamination from pion-related events) is shown as well.

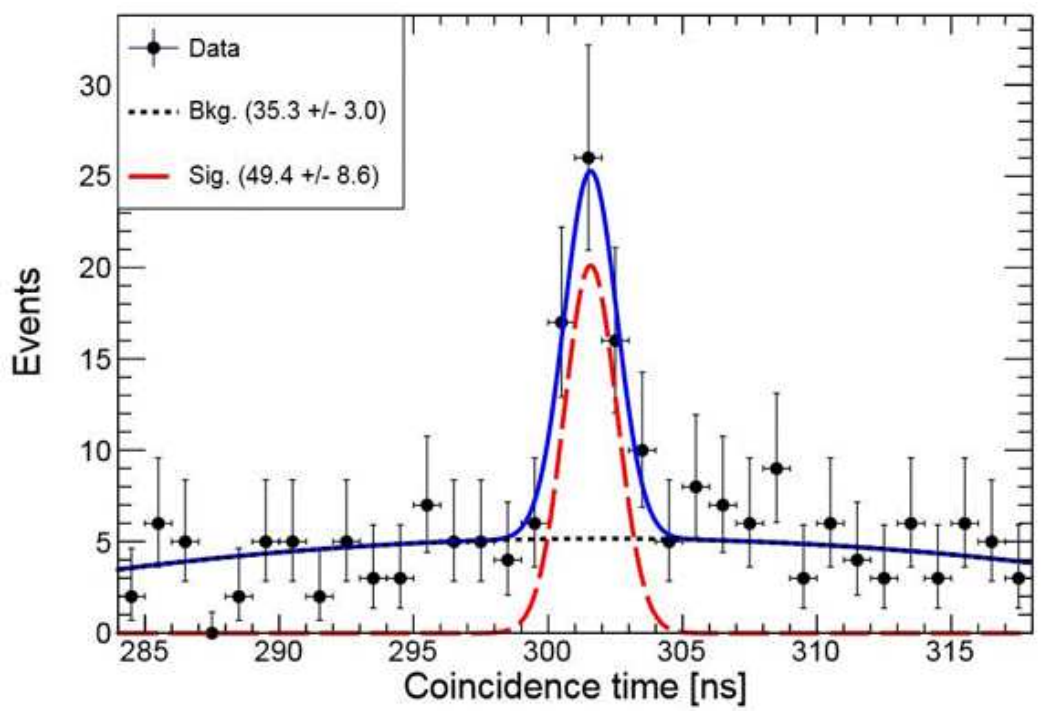

Figure 3: Timing spectra for the $60^{\circ}$ detector showing the coincident peak and the background.

The differential cross section is shown in Figure 4 for the detector at $60^{\circ}$. The data agree with the general expectation that the Compton cross section should increase as the photon energy increases above pion threshold. Unfortunately, the relatively large uncertainties of these new data are too large to make a meaningful extraction of $\alpha_{n}$ and $\beta_{n}$. The data at the back angles produced visible coincident peaks, but the signal-to-noise ratio was too large to extract cross sections with reasonable uncertainties. Those results are not reported here. 


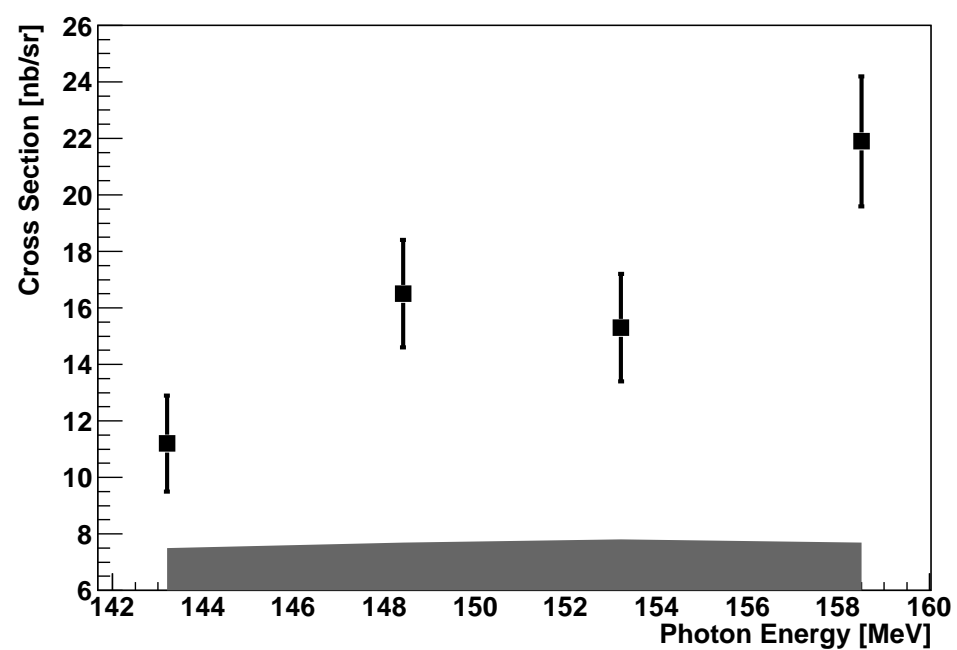

Figure 4: Compton-scattering cross section at $60^{\circ}$ as a function of incident photon energy. Statistical uncertainties are shown. Systematic uncertainties indicated by the gray band.

\section{Conclusion}

This experiment indicates that measurement of the Compton cross section near pion threshold is possible. The major limitation to the results reported here is statistical as the experimental setup was not optimized for collecting Compton-scattering data with high statistics. The analysis indicate near threshold measurements $\left(E_{\gamma} \leq 150 \mathrm{MeV}\right)$ are nearly impossible without an active target. The elastically-scattered photon peak will be contaminated by photons coming from pion interactions. This will be especially problematic at back angles, which could require slightly higher photon energies to extract cross sections. However, at appropriate energies, the measurements can be obtained and, in principle, cross sections and polarizabilities extracted. The success of such future experiments would have multiple benefits: adding to the number of data points in the $d\left(\gamma, \gamma^{\prime}\right) d$ data set, adding data points that are more sensitive the polarizabilities, and creating a data set that is more comparable to the $p\left(\gamma, \gamma^{\prime}\right) p$ data set.

We hope that this work will inspire future experimental and theoretical work on the $d\left(\gamma, \gamma^{\prime}\right) d$ reaction above pion photoproduction threshold.

\section{References}

[1] B. Strandberg, et. al., Compton scattering from the deuteron above pion-production threshold, Phys. Rev. C 98 012201(R) (2018) [nucl-ex/1803.07308]

[2] H.W. Griesshammer, J.A. McGovern, D.R. Phillips, and G. Feldman, Using Effective Field Theory to analyse low-energy Compton scattering data from protons and light nuclei, Prog. Part. Nucl. Phys. 67 841 (2012) [nucl-th/1203.6834]

[3] F. Hagelstein, R. Miskimen, and V. Pascalutsa, Nucleon Polarizabilities: From Compton scattering to hydrogen atom, Prog. Part. Nucl. Phys. 8829 (2016) [nucl-th/1512.03765] 
[4] L.S. Myers, et. al., Measurement of Compton Scattering from the Deuteron and an Improved Extraction of the Neutron Electromagnetic Polarizabilities, Phys. Rev. Lett. 113262506 (2014) [nucl-ex/1409.3705]

[5] A. Walker-Loud, C.E. Carlson, and G.A. Miller, Electromagnetic Self-Energy Contribution to $M_{p} \hat{a} \mathcal{L} \breve{S} M_{n}$ and the Isovector Nucleon Magnetic Polarizability, Phys. Rev. Lett. 108232301 (2012) [nucl-th/1203.0254]

[6] M.C. Birse and J.A. McGovern, Proton polarisability contribution to the Lamb shift in muonic hydrogen at fourth order in chiral perturbation theory, Eur. J. Phys. A48 120 (2012) [hep-ph/1206.3030]

[7] J.-O. Adler et al., The Upgraded Tagging Facility at the MAX IV Laboratory, Nucl. Inst. Meth. A715 1 (2012).

[8] J.-O. Adler et al., A broad range tagging spectrometer for the MAX-laboratory, Nucl. Inst. Meth. A388 17 (1997).

[9] J.M. Vogt et al., The Photon Tagging Facility at the Saskatchewan-Accelerator-Laboratory, Nucl. Instr. and Meth. A339 425 (1994). 